\title{
Film thickness measurements in liquid-liquid slug flow regimes
}

\author{
Marc Mac Giolla Eain ${ }^{a, *}$, Vanessa Egan ${ }^{a}$, Jeff Punch ${ }^{b}$ \\ a Stokes Institute, Mechanical Aeronautical \& Biomedical Engineering, University of Limerick, Limerick, Ireland \\ ${ }^{\mathrm{b}}$ CTVR, Stokes Institute, Mechanical Aeronautical \&' Biomedical Engineering, University of Limerick, Limerick, Ireland
}

\section{A R T I C L E I N F O}

\section{Article history:}

Received 9 August 2012

Received in revised form 14 August 2013

Accepted 19 August 2013

Available online 13 September 2013

\section{Keywords:}

Multiphase flow

Liquid-liquid flow

Slug flow

Film thickness

Slug length

\begin{abstract}
A B S T R A C T
At present there is significant interest in the development of small scale medical diagnostic equipment. These devices offer faster processing times and require smaller sample volumes than equivalent macro scale systems. Although significant attention has been focused upon their outputs, little attention has been devoted to the detailed fluid mechanics that govern the flow mechanisms within these devices. Conventionally, the samples in these small scale devices are segmented into distinct discrete droplets or slugs which are suspended in an organic carrier phase. Separating these slugs from the channel wall is a very thin film of the organic carrier phase.

The magnitude of this film is the focus of the present study and the effects of sample slug length and carrier phase fluidic properties on the film are examined over a range of Capillary numbers. A non-intrusive optical technique was used to capture images of the flow from which the magnitude of the film was determined.

The experimental results show that the film is not constant along the length of the slug; however above a threshold value for slug length, a region of constant film thickness exists. When compared with existing correlations in the literature, the experimental data showed reasonable agreement with the Bretherton model when the Capillary number was calculated based on the mean two phase flow velocity. However, significant differences were observed when the Capillary number was redefined to account for the mean velocity at the liquid interface, i.e., the mean slug velocity.

Analysis of the experimental data revealed that it fell into two distinct flow regimes; a visco-capillary regime and a visco-inertial regime. A modified Taylor expression is presented to estimate the magnitude of the film for flows in the visco-capillary regime while a new model is put forward, based on Capillary and Weber numbers, for flows in the visco-inertial regime. Overall, this study provides some novel insights into parameters, such as aqueous slug length and carrier phase fluidic properties, that affect the thickness of the film in liquid-liquid slug flow regimes.
\end{abstract}

(ㄷ) 2013 Published by Elsevier Inc.

\section{Introduction}

Multiphase flow systems offer numerous advantages over traditional single phase flow systems and as a result are utilised in most emerging technologies that employ micro fluidics. Multiphase flow systems can be found in a diverse range of technologies including high heat flux removal systems (Howard et al., 2011), chemical engineering (Tice et al., 2003) and monolith reactors (Kreutzer et al., 2005). However, one of the most promising applications of multiphase flows is in biotechnological devices such as biochips and biosensors. Use of multiphase flows, or, more specifically liquid-liquid multiphase flows, offers an alternative approach to the processing of large numbers of chemical or biological samples. This approach, where chemical or biological samples are separated into distinct separate streams of slugs or droplets and are sepa-

\footnotetext{
* Corresponding author. Tel.: +353 61233619; fax: +353 61202393 .

E-mail address: marc.macgiollaeain@ul.ie (M. Mac Giolla Eain).
}

rated from one another by a segmenting fluid offers a host of advantages over single phase continuous flow methodology. Such benefits include enhanced mixing within samples, high throughput levels, reduced analysis times, reusability of devices and reduction in the cost of consumables.

Separating these chemical or biological samples from the channel walls is a very thin liquid film of the continuous phase. This film protects the samples from any asperities within the channels and aids in the prevention of cross contamination between samples. The presence and magnitude of the film also has considerable effects on both the heat (Howard et al., 2011), and mass, (Ghaini et al., 2010), transfer rates. The focus of the present study is the magnitude of the film that exists in liquid-liquid slug flow regimes. Numerous researchers have completed numerical, (Araujo et al., 2012; Heil, 2001; Taha and Cui, 2004) and experimental, (Grimes et al., 2007; Irandoust and Andersson, 1989; Jovanovic et al., 2010) analysis of the deposition of the liquid film on the capillary wall. Some of these works have resulted in the development of a 


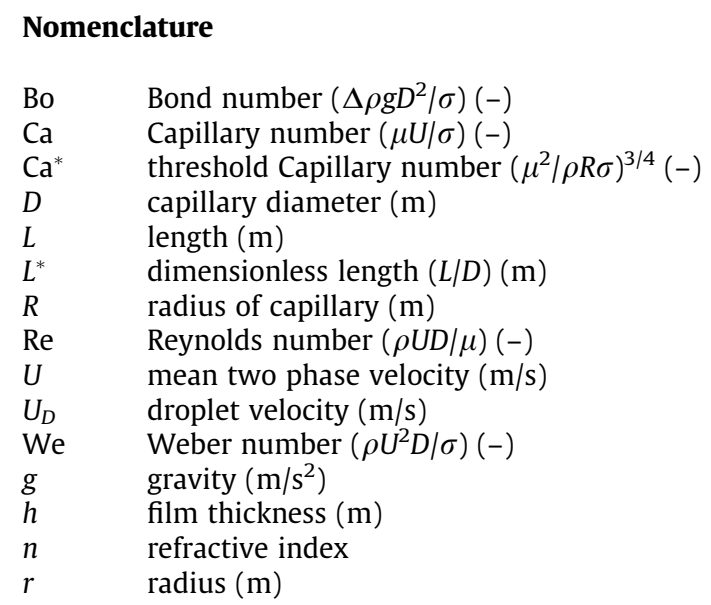

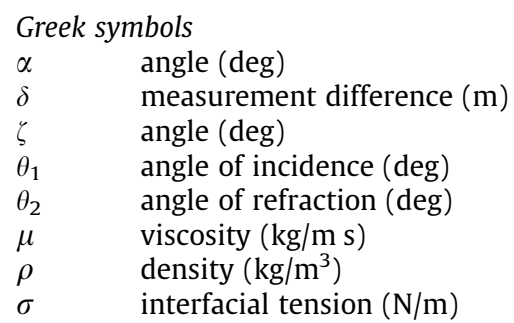

$\begin{array}{ll}\text { Subscripts } \\ \text { A } & \text { apparent } \\ \text { Act } & \text { actual } \\ C & \text { continuous oil phase } \\ D & \text { discontinuous water phase }\end{array}$

number of models to estimate the magnitude of the film; the details of the most referenced of these are listed in Table 1 . In their pioneering work (Fairbrother and Stubbs, 1935), while examining liquid flow rates in capillaries using an indicator bubble, noted that the bubble moved faster than the liquid and concluded that this was due to the presence of a liquid film surrounding the bubble. Taylor (1960) using a similar methodology measured the magnitude of this film by comparing the difference between the bubble and mean flow velocity.

Aussillous and Quere (2000) developed an empirical correlation based on the data of Taylor and found a dependence of the normalised film thickness on a single parameter, namely the Capillary number. This correlation became known as Taylor's Law, Table 1, where the coefficient 1.34 was derived by Bretherton (1960) and the coefficient 2.5 is empirical and valid up to $\mathrm{Ca}=1.4$. Aussillous and Quere (2000) noted that at higher deposition velocities, Taylor's Law under predicted the thickness of the film due to the emergence of a visco-inertial regime. Further increases in velocity resulted in a reduction in the rate of film growth due to tube confinement, as a threshold Capillary number had been reached. Grimes et al. (2007) examined the validity of the Taylor and Bretherton laws when applied to liquid-liquid flows and found that these expressions under predicted the thickness of the film. The authors redefined the Capillary number to include the viscosity of the slug rather than the carrier and found the data to collapse onto a single curve with little scatter.

Han and Shikazono (2009) studied both the local and instantaneous film thickness in liquid-gas flows using an interferometer and laser focus displacement meter. Examining the flow over a wide range of Reynolds and Capillary numbers, they found that at low values of Capillary number inertial forces could be neglected and that Capillary number alone could be used to determine film thickness. However, with increasing Capillary number, an increase in Reynolds number initially resulted in a reduction in film thick- ness to a minimum value before subsequently increasing again. Similar observations were made by Heil (2001) and to account for these increased inertial effects (Han and Shikazono, 2009) developed the expression in Table 1 , which incorporates both the Reynolds and Weber numbers.

The thickness of the film present in liquid-gas flows has received significant attention in the literature and has been the subject of numerous studies (Giavedoni and Saita, 1999; Goldsmith and Mason, 1963; Heil, 2001; Howard and Walsh, 2013; Martinez and Undell, 1990). While numerous applications employ liquid-liquid flow regimes, little is known about the thickness of the film associated with such a flow regime. This paper aims to address this deficit in the literature by measuring the thickness of the film in a liquid-liquid slug flow regime. A segmented liquid-liquid slug flow regime was created that consisted of aqueous slugs dispersed in a continuous carrier oil. The carrier oil was varied, while the discontinuous phase remained constant through-out. The variations in the physical properties of the carrier phases resulted in the desired range of Capillary number, 0.002-0.119. Non-dimensional film thickness values were determined from a series of images captured using an optical technique and the effects of aqueous slug length and carrier phase on film thickness were examined. Comparisons are made between the experimental data and the most referenced expressions in the literature and a number of recommendations are made as to their use and applicability.

\section{Experimentation}

In order to measure the magnitude of the film that exists between the aqueous slugs and the capillary wall, a custom measurement facility was designed and constructed that allowed a non-invasive measurement of the film. A description of the bulk experimental setup, carrier phases used, optical corrections necessary and an uncertainty analysis are presented in this section.

Table 1

Correlations from literature.

\begin{tabular}{|c|c|c|}
\hline Author & Correlation & Parameters \\
\hline Fairbrother and Stubbs (1935) & $\frac{h}{R}=0.5 \mathrm{Ca}^{1 / 2}$ & $7.5 \times 10^{-5}<\mathrm{Ca}<0.014$ Liquid-gas flows horizontal flow single slug \\
\hline Bretherton (1960) & $\frac{h}{R}=1.34 \mathrm{Ca}^{2 / 3}$ & $\mathrm{Ca}<0.003$ Liquid-gas flows horizontal flow single slug \\
\hline Aussillous and Quere (2000) & $\frac{h}{R}=\frac{1.34 \mathrm{Ca}^{2 / 3}}{1+1.34\left(2.5 \mathrm{Ca}^{2 / 3}\right)}$ & $0.015<\mathrm{Ca}<1.9$ Liquid-gas flows vertical flow single slug \\
\hline Han and Shikazono (2009) & $\frac{h}{R}=\frac{1.34 \mathrm{Ca}^{2 / 3}}{1+3.13 \mathrm{Ca}^{2 / 3}+0.54 \mathrm{Ca}^{0.672} \mathrm{Re}^{0.589}-0.352 \mathrm{We}^{0.629}}$ & $\mathrm{Ca}<0.40$ Liquid-gas flows horizontal flow multiple slugs \\
\hline
\end{tabular}




\subsection{Bulk measurement setup}

A schematic of the experimental apparatus that was used to measure the magnitude of the film is shown in Fig. 1. Two precision Harvard PhD 2000 programmable syringe pumps were used to set the volumetric flow rates. Separate syringe pumps allowed the relative flow rates, and hence slug lengths to be varied over a wide range. The liquid phases were delivered by $100 \mathrm{~mL}$ capacity Hamilton 1100TLL gas tight glass syringes. Glass syringes were used as they deliver steadier flow rates than those attainable from plastic syringes.

Two methods were used to generate the aqueous slugs over the course of this study: T-junctions and segmenters. Segmenters, as shown in Fig. 2, work by periodically creating and rupturing a liquid bridge between two opposing capillary tips and have been used previously by Curran et al. (2005) to dispense sub-micro litre volumes of reagents to be used as microfluidic chemical reactors in the PCR process. The water forms a droplet at the tip of the inlet capillary such that, when sufficiently large, it forms a stable liquid bridge between the opposing capillary. Fluid is drained from the liquid bridge until it reaches the minimum volume stability limit and ruptures, dispensing a water slug. This cycle is then repeated creating a chain of water slugs punctuated by oil. Segmenters require the reservoir to be filled with a density matched oil to ensure a buoyancy free environment exists for axisymmetric liquid bridg- ing. As a result, only the Pd5/water slug flows were generated through segmentation. For all other oil/water combinations examined in this study, T-junctions were used, where the formation of the aqueous slugs is driven by the shear force exerted by the carrier phase on the dispersed phase.

Once generated, the aqueous slug train proceeded to the main capillary which was horizontal in orientation and consisted of transparent Upchurch Scientific ${ }^{\circledR}$ FEP (fluorinated ethylene propylene) Teflon tubing. The tubing was approximately $1 \mathrm{~m}$ in length and had an internal diameter of $1.59 \mathrm{~mm}$. The flow was imaged at a single location $0.75 \mathrm{~m}$ downstream from the entrance of the main capillary; this was to ensure the full hydrodynamic development of the flow prior to imaging. The region being imaged was immersed in a transparent water bath. The refractive index of water is 1.33, (Meinhart et al., 1999), while the refractive index of the tubing, according to the manufacturer, is 1.338 . This almost perfect refractive index matching resulted in excellent visualisation of the flow. Images of the flow were captured using an IDT X-Stream XS-4 CMOS high-speed camera mounted on a Zeiss Axioskop microscope. Images were recorded using $1.5 \times$ and $20 \times$ lenses at a frequency of $5000 \mathrm{~Hz}$ ( 1 image every $200 \mu \mathrm{s}$ ), and an exposure of $122 \mu \mathrm{s}$. Images captured using the $20 \times$ lens were analysed in Matlab (version 2010a), where a custom code extracted measurements of liquid film thickness along the length of the aqueous slug. While images captured using the $1.5 \times$ lens were

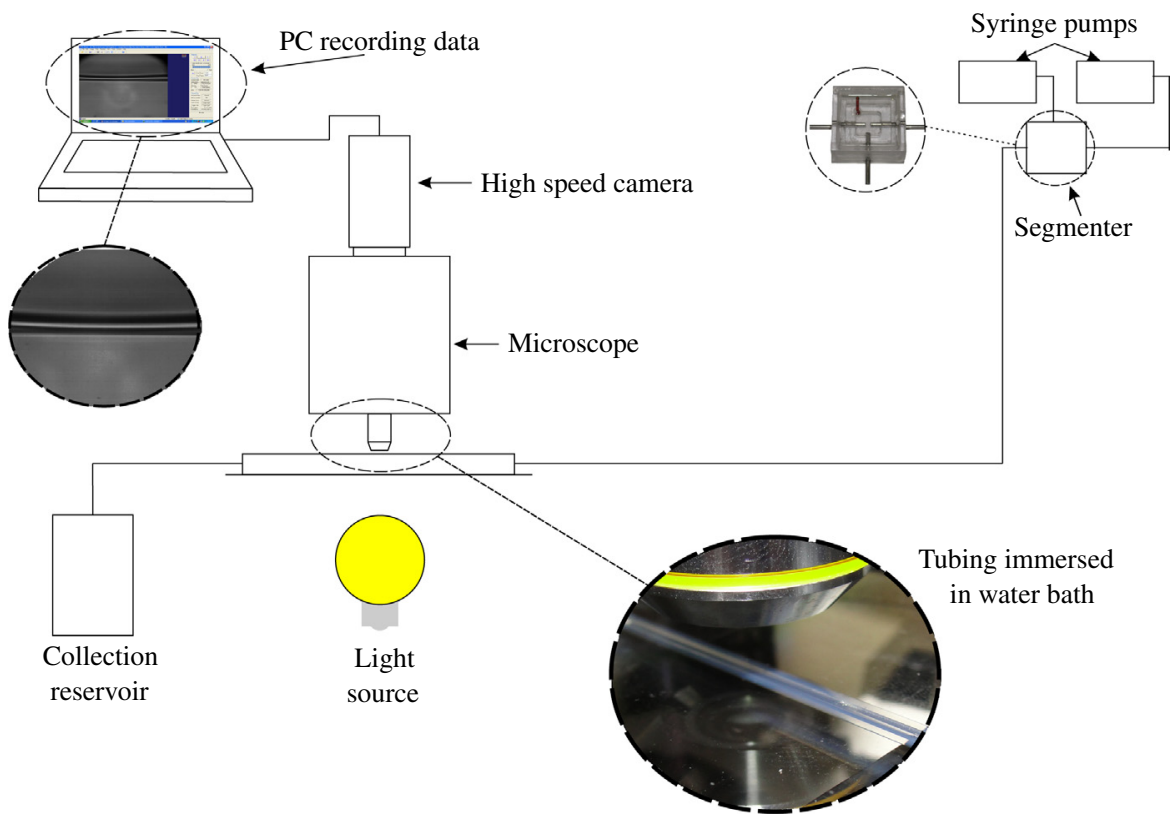

Fig. 1. Schematic illustration of experimental test facility with key features highlighted.

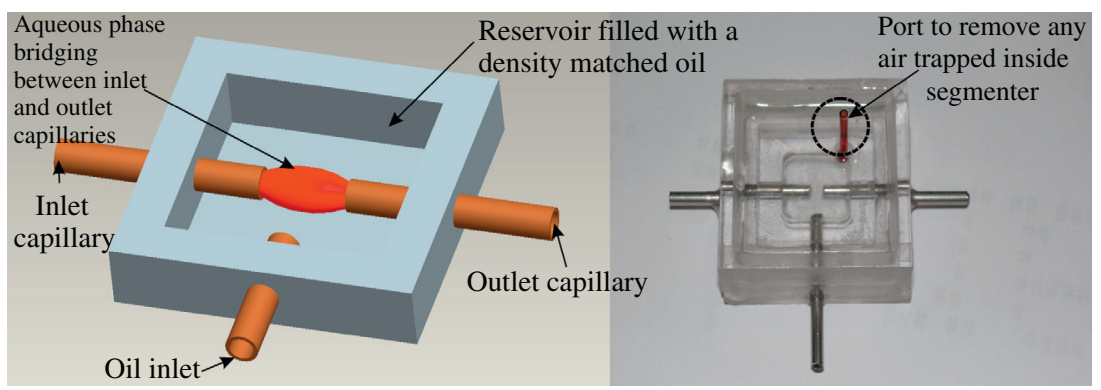

Fig. 2. Segmentation device used in the generation of liquid-liquid slug flow regime. 
Table 2

Thermophysical properties of the fluids used in study, recorded at room temperature and atmospheric pressure.

\begin{tabular}{lclll}
\hline Fluid & $\begin{array}{l}\text { Density } \\
\left(\mathrm{kg} / \mathrm{m}^{3}\right)\end{array}$ & $\begin{array}{l}\text { Viscosity }(\mathrm{kg} / \\
\mathrm{m} \mathrm{s})\end{array}$ & $\begin{array}{l}\text { Interfacial tension } \\
(\mathrm{N} / \mathrm{m})\end{array}$ & $\begin{array}{l}\text { Refractive } \\
\text { index }\end{array}$ \\
\hline Water & 997.1 & 0.00091 & - & 1.330 \\
Pd5 & 911.8 & 0.00358 & 0.0397 & 1.438 \\
Dodecane & 754.3 & 0.00139 & 0.0520 & 1.422 \\
FC40 & 1854.1 & 0.00330 & 0.0511 & 1.290 \\
AR20 & 1142.2 & 0.02091 & 0.0302 & 1.440 \\
\hline
\end{tabular}

used to determine aqueous slug length, spacing and velocity. For any specific test, the distance travelled by a slug over a series of images could be measured and by knowing the rate at which the images was recorded, the slug velocity could be determined.

\subsection{Continuous carrier phase}

In the current study, experiments were carried out using a selection of different carrier fluids, while water was used as the discontinuous phase in all tests. Four carrier oil/water combinations were examined allowing the effects of a wide range of physical properties on film thickness to be evaluated. The four carrier oils used were; Pd5 baysilone, Dodecane and AR20 silicone oils and FC40 fluorinert fluid. Physical properties of the different liquid mediums are presented in Table 2. Density was determined by measuring the mass of $1 \mathrm{~mL}$ of each fluid. Viscosities were measured over a range of temperatures using a Brookfield LVDV-II+ viscometer and calibration bath. The interfacial tension between the oil and water phases was measured using the pendant drop method (Saad et al., 2011). Refractive index values for each fluid were provided by the manufacturers. Table 3 presents the range in non-dimensional parameters encountered in the current study. The Reynolds, Capillary and Weber numbers span several orders of magnitude while the dimensionless oil and aqueous slug lengths span two orders of magnitude. These values are typical of those encountered in most micro and mini scale fluidic systems.

\subsection{Optical corrections}

While the proposed measurement technique provides a straight forward and non-invasive measurement of film thickness, there are however, a series of post processing steps required, due to the refraction of the light through the liquid phases, to determine the true liquid film thickness. As light rays pass through the capillary wall, they are scattered due to changes in refractive index. These effects are corrected using a series of trigonometric operations coupled with the geometrical optics of light refraction. Fig. 3 graphically illustrates the effects of refractive index changes on the light rays. As the tubing and surrounding water have an almost identical refractive index, it is assumed that the refraction of the light through the tubing can be neglected and the position of the capillary wall seen in the images is the true position of the cap-

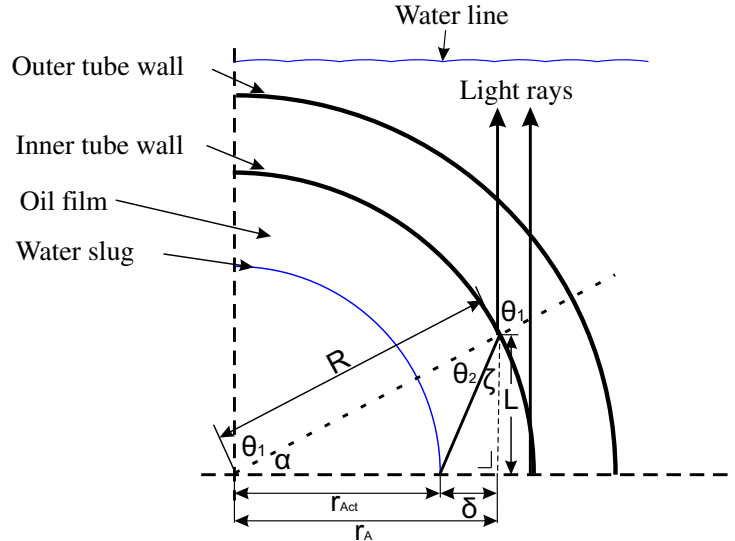

Fig. 3. Schematic illustration of technique used to correct for refractive index mismatches.

illary wall. However, depending on the refractive index of the carrier phase, the visible film will be larger or smaller than the actual film. A measure of the apparent film thickness is taken and from this the apparent slug radius $\left(r_{A}\right)$ is inferred. This allows the angle $\alpha$ to be determined and from this $\theta_{1}$, the angle the light ray makes with the inner wall of the capillary and an axis normal to the surface.

$\cos \alpha=\frac{r_{A}}{R}$

$\theta_{1}=90-\alpha$

Use of Snell's law allows the refracted angle, $\theta_{2}$, to be solved.

$n_{1} \sin \theta_{1}=n_{2} \sin \theta_{2}$

where $n_{1}$ and $n_{2}$ are the refractive indices of the tubing and the carrier phase respectively. The difference between the apparent and actual film thickness can now be solved using a series of simple trigonometric relations. The length $L$ is calculated using Pythagoras' theorem and the angle $\zeta$ is simply the difference between the angles $\theta_{1}$ and $\theta_{2}$.

$L=\sqrt{R^{2}-r_{A}^{2}}$

$\delta=(L) \tan \left(\theta_{1}-\theta_{2}\right)$

The actual aqueous slug radius, $\left(r_{A c t}\right)$, is then solved and from this the actual film thickness is determined.

$r_{\text {Act }}=r_{A}-\delta$

\subsection{Uncertainty analysis}

The uncertainty in the resolution of a pixel was \pm 1 , which resulted in the uncertainty in measured distance of $\pm 3 \mu \mathrm{m}$, uncertainty in the tabulated fluid properties was $\pm 1 \%$. Using these values, the uncertainties in slug velocity, Capillary number and

Table 3

Range of dimensionless experimental parameters considered in this study.

\begin{tabular}{|c|c|c|c|c|}
\hline Oil/water combination & Pd5/water & Dodecane/water & FC40/water & AR20/water \\
\hline$L_{D}^{*}$ & $2.18-8.11$ & $1.47-4.71$ & $1.16-3.61$ & $0.87-3.45$ \\
\hline$L_{C}^{*}$ & $1.88-14.14$ & $1.11-8.52$ & $0.72-8.62$ & $0.58-4.02$ \\
\hline $\mathrm{Ca}$ & $0.003-0.011$ & $0.002-0.003$ & $0.002-0.007$ & $0.029-0.119$ \\
\hline $\mathrm{Ca}^{*}$ & 0.003 & 0.0007 & 0.001 & 0.126 \\
\hline $\operatorname{Re}$ & $14.46-47.53$ & $61.952-95.86$ & $31.02-100.96$ & $3.68-15.13$ \\
\hline We & $0.047-0.518$ & $0.283-0.119$ & $0.073-0.697$ & $0.106-1.671$ \\
\hline Bo & 0.053 & 0.115 & 0.414 & 0.118 \\
\hline
\end{tabular}


film thickness were calculated using the method of Kline and McClintock (1953), were: $U_{s}: \pm 2.1 \%$, Ca: $2.92-5.96 \%$ and $h$ : $2.17-$ $10.87 \%$.

\section{Results and discussion}

The following section presents and analyses the experimentally measured film thickness data. Results are determined from a series of images captured using a high speed camera attached to a microscope. Each slug image presented in the following section is a compilation of a series of images stitched together. Due to the low Bond numbers associated with the flows, see Table 3, gravitational effects were assumed to be negligible and as a result the flow was assumed axisymmetric. The results determined from the experimental measurements are presented and discussed in two separate sub-sections that examine: the formation of the liquid film and its variation with aqueous slug length and the predictive models used to estimate the magnitude of the film.

\subsection{Film formation and variation}

A segmented liquid-liquid flow regime is generated when two immiscible liquids are pumped into a channel over a range of flow rates. The generated flow regimes are the result of surface and body force interactions and include, but are not limited to: churn, annular, slug, plug, stratified and wavy flow. At the micro scale the dominance of surface forces over gravitational forces results in slug and plug flow regimes being the most commonly encountered. In these regimes, the liquid slug occupies the majority of the capillary cross-section leaving only a very thin film of the liquid separating the slug from the capillary wall, as shown in Fig. 4.

The film is formed due to viscous effects at the solid-liquid interface; however, the magnitude of the film depends on a number of parameters including the capillary, inertial and body forces. Examining the motion of bubbles in tubes (Bretherton, 1960) divided a slug into several regions, shown here schematically in Fig. 4. At the front and rear of the slug are hemispherical caps whose shape is maintained by a balance between the uniform pressure within the slug and interfacial tension. Moving towards the centre of the slug, these caps pass through a transition region and proceed to a region where a uniform film of fluid is present.

Numerous authors (Han and Shikazono, 2009; Olbricht and Kung, 1992; Taha and Cui, 2004) have presented slug profiles similar to the one described by Bretherton (1960) in his seminal paper. In these studies, the slugs were of sufficient length to allow the caps to transition into a flat film region. Fig. 5 presents a series of images of the FC40/water flows from the present study that were captured using a $20 \times$ lens. These images were captured at a constant Capillary number of 0.0072 , therefore the changes in slug profile, and hence film thickness, are purely due to changes in slug length rather than a combined effect with other parameters, namely viscous, inertial and interfacial forces. For shorter slug lengths, Fig. 5(a), $L_{D}^{*}=1.15$, no region of uniform film thickness exists. The transition from the front cap continues directly to the rear cap and the profile varies along the entire length of the slug. However, as slug length increases, Fig. 5(b), a change in slug profile is observed. The transition region at the front of the slug increases in length, while at the tail; a wave disturbance is seen prior to the rear cap. This change in slug profile at the tail has also been noted by Goldsmith and Mason (1963) and Olbricht and Kung (1992)) and has been described by Bretherton (1960) as the transition from the body of the slug to the rear cap. Fig. 5(b) shows that the front and rear transition regions intersect, however, no flat film region exists along the slug. A further increase in slug length, as shown in Fig. 5(c), results in a slug profile similar to that described by Bretherton (1960), shown in Fig. 4, where a region of constant film thickness exists.

Similar images to those presented in Fig. 5 were recorded for all carrier/water combinations examined and revealed similar trends in film thickness for changes in aqueous slug length. Analysis of the experimental data, over the range in Capillary and Reynolds numbers examined in this study, has resulted in the development of the following thresholds for changes in slug profile with changes in slug length. For $L_{D}^{*} \leqslant 1.25$ and $1.25 \leqslant L_{D}^{*} \leqslant 1.86$, the slug profile varies from nose to tail, however, for the latter limits, distinct transition regions are present at the front and rear of the slugs. Once the dimensionless slug length exceeds $L_{D}^{*} \geqslant 1.86$, a flat film region has formed along the length of the slug and separating this flat film region from the capillary wall is a film of uniform thickness.

While the slug profile varies with length, the effects of this variation on film thickness are minimal. Fig. 6 is a non-dimensional plot of film thickness against slug length. It can be seen in Fig. 6 that at $L_{D}^{*}<2$, the magnitude of the film varies with slug length. At $L_{D}^{*}<1.86$, the profile of the slug is still developing and no flat film region has formed along the length of the slug. As a result, the magnitude of the film was measured within the transition regions that exist between the hemispherical caps, as shown in Fig. 5(a) and (b). Consequently, it is proposed that the location of measurement, resulting from the nonexistence of a flat film region, is the cause of this variation in film thickness with slug length. However, once $L_{D}^{*}>1.86$, a flat film region forms, and, similar to observations presented by Han and Shikazono (2009) and Howard and Walsh (2013), variations in film thickness with slug length are minimal and it is believed that these variations fall within experimental uncertainty, hence any effects of slug length on film thickness cannot be clearly identified.

\subsection{Film thickness models}

The previous sub-section revealed that slug profiles and hence film thickness vary along the length of the slug. This makes the

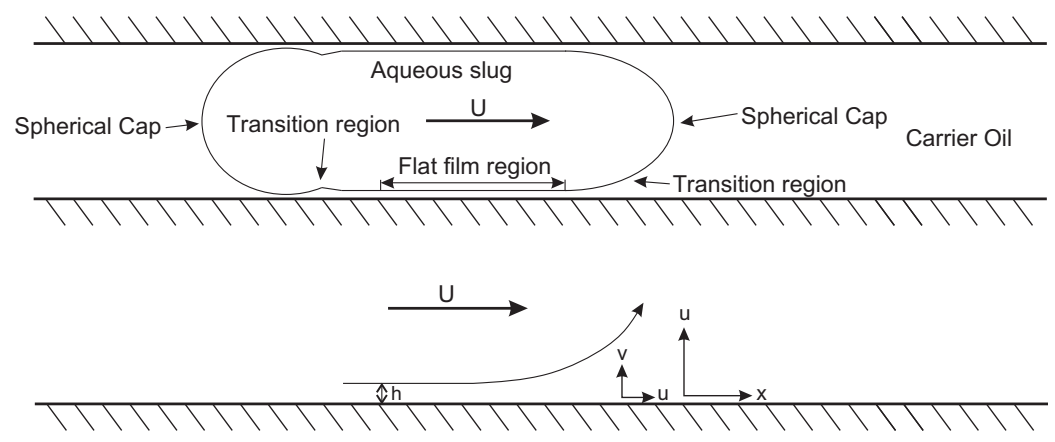

Fig. 4. Illustration highlighting the different regions within a slug. 
(a)
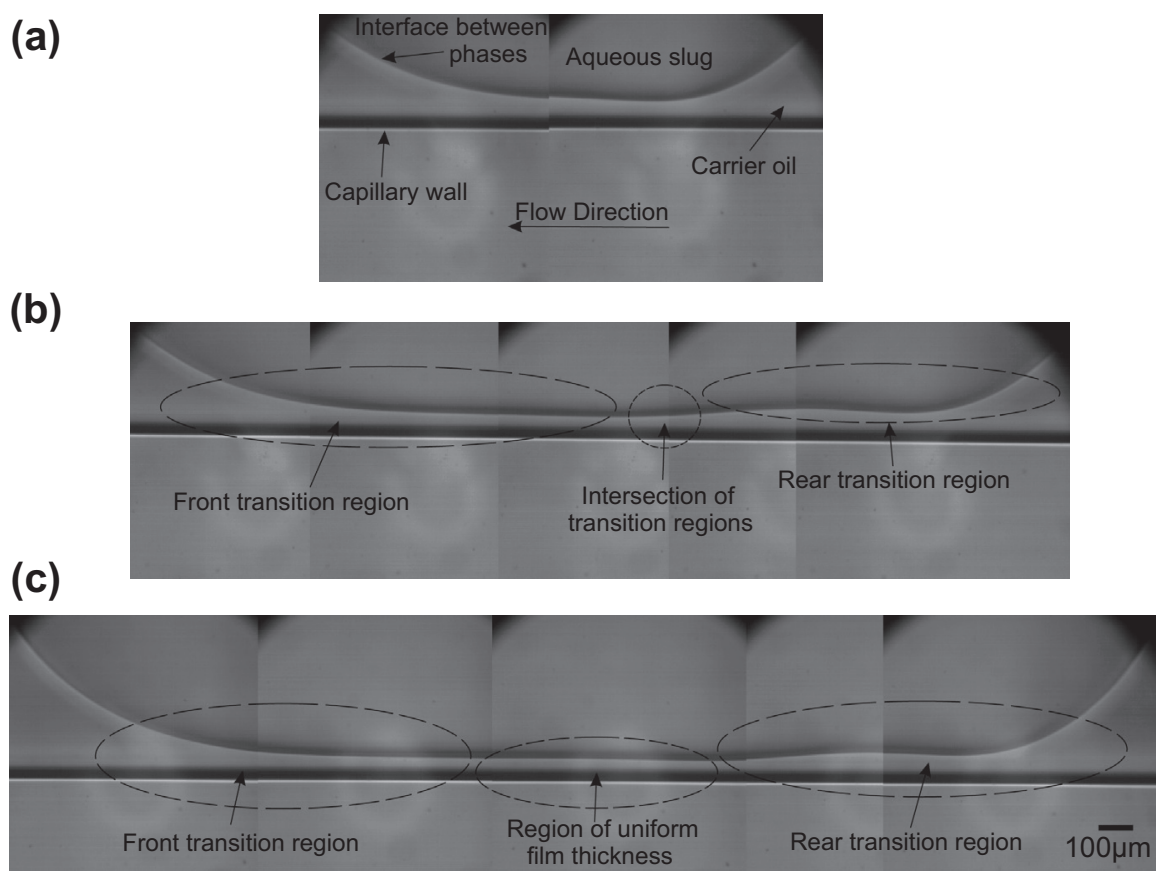

Fig. 5. Experimental images of FC40/water flow, flowing at $\mathrm{Ca}=0.0072$, highlighting the variation in slug profile with length at $L^{*}$ of (a) 1.15 , (b) 1.81 and (c) 2.76 .

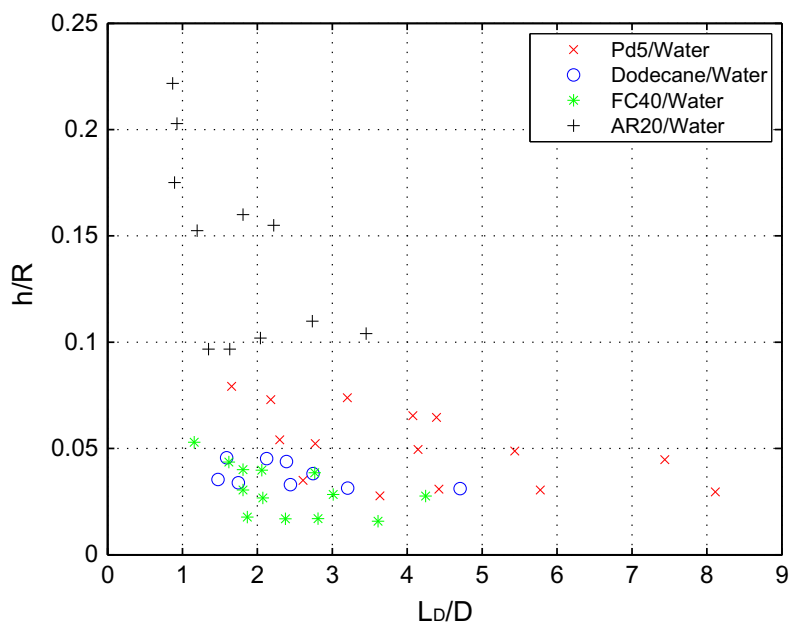

Fig. 6. Non-dimensional plot of film thickness, $h / R$, against slug length, $L_{D} / D$.

choice of location for film thickness measurement difficult. Some authors report film measurements based on the thickness at the flat section midway between the front and rear caps (Han and Shikazono, 2009; Jovanovic et al., 2010). However, this is only possible when slug lengths are greater than the previously defined threshold of $L_{D}^{*} \geqslant 1.86$. Other authors have overcome this variation in film thickness with slug length by using indirect measurement techniques (Grimes et al., 2007; Irandoust and Andersson, 1989; Kashid et al., 2005; Taylor, 1960), that give an average of the film thickness along the length of the slug. Where possible, the experimental data presented in this sub-section was measured at the flat film region. However, for slugs where $L_{D}^{*} \leqslant 1.86$, the measurement was taken towards the rear of the slug. This location was chosen as, similar to the cases where the flat film region was present; the slug was at its broadest hence the film was at its thinnest.

Previous works (Aussillous and Quere, 2000; Fairbrother and Stubbs, 1935) have shown that film thickness is highly dependent on Capillary number. Fig. 7 is a plot of the non-dimensional film thickness, $h / R$, for each of the carrier oil/water combinations examined in this study against Capillary number. Included in the plot is an enlarged view of the data in the lower Capillary number region, $\mathrm{Ca} \leqslant 0.01$. Experimental data points are compared with the (Aussillous and Quere, 2000; Bretherton, 1960; Fairbrother and Stubbs, 1935; Han and Shikazono, 2009) expressions. The experimental data shows reasonable agreement with the Bretherton model, with data points estimated by the model falling within $\pm 25 \%$ of the experimentally measured data points. However, the other models greatly underestimate the magnitude of the film; this is particularly evident for $\mathrm{Ca} \geqslant 0.009$.

While the data presented in Fig. 7 shows a strong dependence of film thickness on Capillary number, there is however, a problem with the definition of the Capillary number. The Capillary numbers plotted in Fig. 7 are defined using the viscosity of the wetting phase, the mean two phase velocity and the interfacial tension between the two liquids. The problem with this definition of Capillary number is the velocity term used. As stated previously in Section 3.1, the magnitude of the film depends on a number of different parameters, including the velocity at the interface between the phases, i.e., the mean slug velocity. At low Capillary numbers this is approximately equal to the mean two phase velocity, however, as the Capillary number increases, the film increases in thickness, and, as a result, the slug velocity increases. Mean slug velocities were calculated using the images captured with the $1.25 \times$ lens and from which corresponding Capillary numbers were calculated. This redefined Capillary number, unlike the Capillary number based on the mean two phase velocity, provides a truer representation of the forces influencing the magnitude of the film at the point of measurement. Fig. 8 is a plot of non-dimensional film thickness against the redefined Capillary number. Included in Fig. 8 is an enlarged view of the data in the lower Capillary number range, $\mathrm{Ca} \leqslant 0.01$.

A study by Aussillous and Quere (2000) on film thickness in slug flows revealed the presence of two distinct flow regimes: a viscocapillary regime and a visco-inertial regime. In a visco-capillary flow regime the normalised film thickness is dependent on a single parameter, the Capillary number, and can be estimated using the 


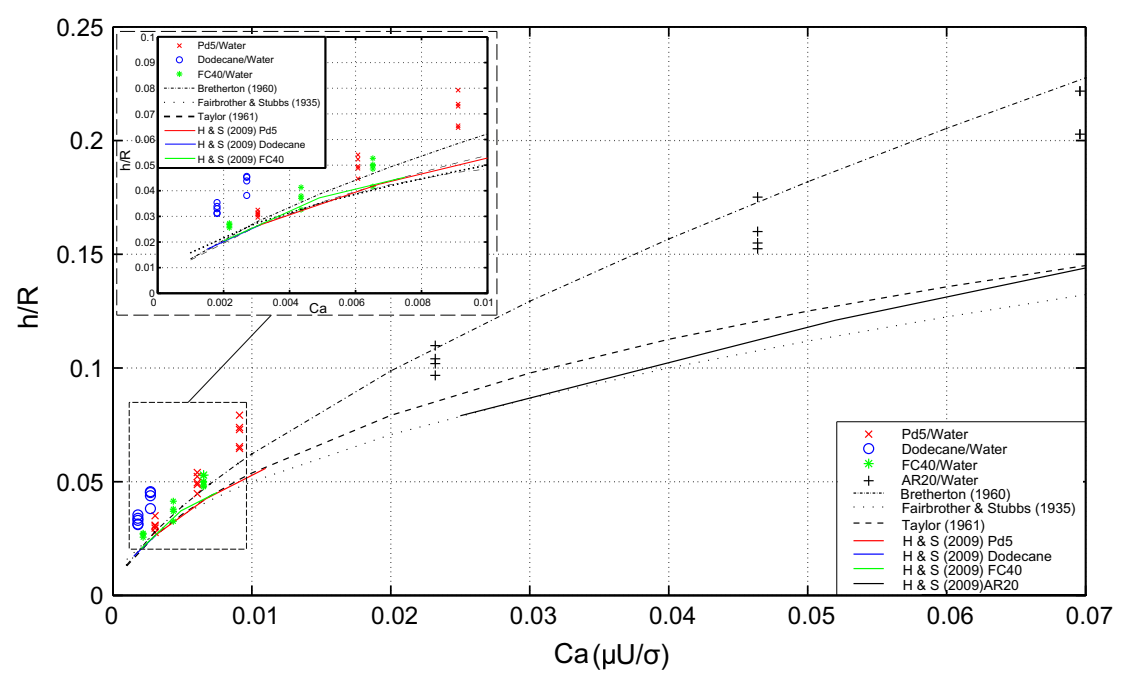

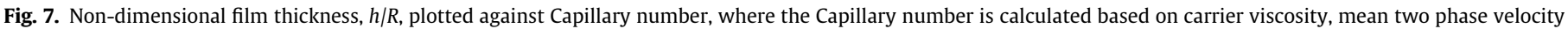
and interfacial tension between the phases. Experimental results are compared with the most referenced correlations in the literature, see Table 1.

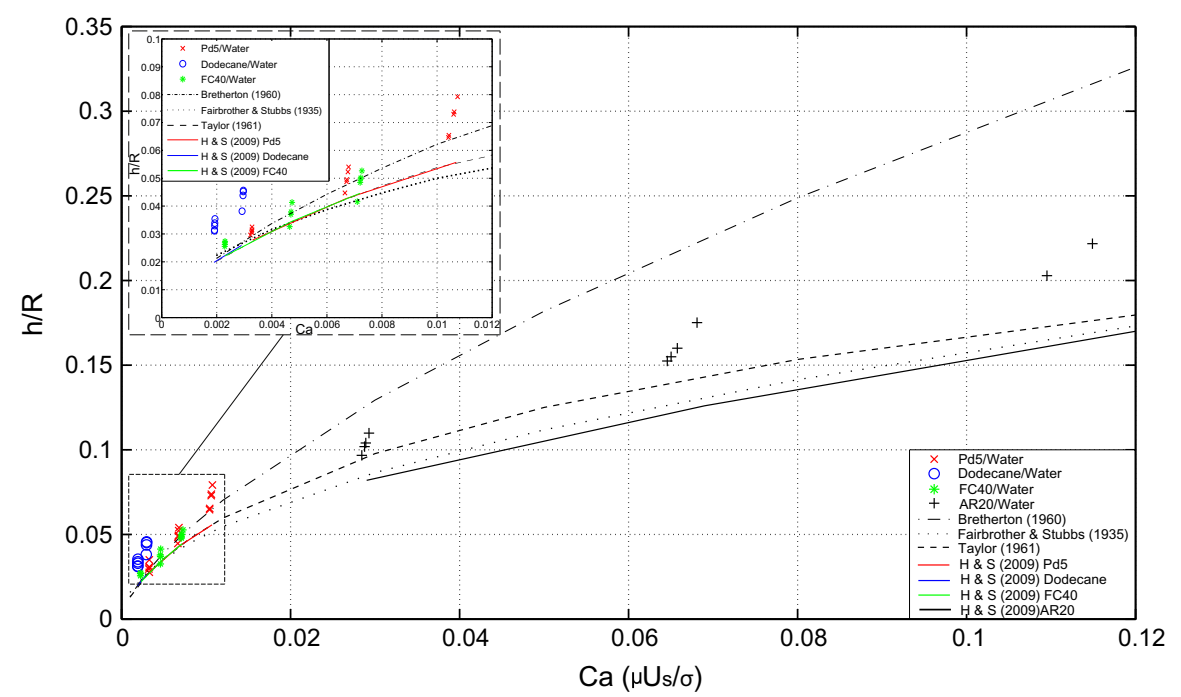

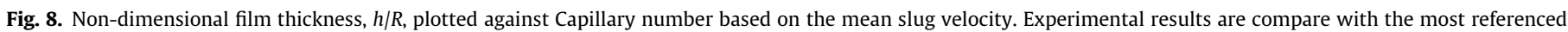
correlations in the literature, presented in Table 1.

Taylor's Law, see Table 1. This expression was developed using an array of carrier oils of differing viscosities to achieve a wide range in Capillary numbers. Flows in the visco-capillary regime are characterised by low deposition velocities resulting in minimal inertial effects. Increases in deposition velocity result in a transition from the visco-capillary regime to a visco-inertial regime. In a viscoinertial regime the flows are subject to increased inertial and reduced viscous effects. Aussillous and Quere (2000) noted that this transition occurred at $\mathrm{Ca}^{*}$, presented in Eq. (7), which is based on the carrier viscosity, carrier density, capillary radius and interfacial tension.

$\mathrm{Ca}^{*} \sim\left(\frac{\mu^{2}}{\rho R \sigma}\right)^{3 / 4}$

$\mathrm{Ca}^{*}$ values for the flows examined in this study are presented in Table 3. Measurements from the current study show that the AR20/ water flows fall into the visco-capillary regime, with $\mathrm{Ca}<\mathrm{Ca}^{*}$ and $\operatorname{Re}<15$, meaning inertial forces have minimal effects on the magni- tude of the film. As seen in Fig. 8 the AR20/water measurements follow a similar trend to the model of Aussillous and Quere (2000), however, this model under predicts the magnitude of the film, particularly as the Capillary number increases, $\mathrm{Ca}>0.03$. Aussillous and Quere (2000) developed an empirical correlation based on the data of Taylor (1960) in which they modified Bretherton's lubrication theory to account for flows where the film occupies a significant portion of the channel. In the present study, the film present in the AR20/water flows occupies between $10 \%$ and $22 \%$ of the channel. The empirical constant in this correlation, presented in Table 1 , is stated as 2.5 , however for the data presented in the current study on liquid-liquid flows, a value of 1.6 was found to give better agreement with the experimental data, within $\pm 10 \%$. A modified Taylor's Law for liquid-liquid flows in the visco-capillary regime is presented in Eq. (8), and, for completeness is plotted in Fig. 9 against the AR20/water data.

$$
\frac{h}{R}=\frac{1.34 \mathrm{Ca}^{\frac{2}{3}}}{1+1.34\left(1.6 \mathrm{Ca}^{\frac{2}{3}}\right)}
$$




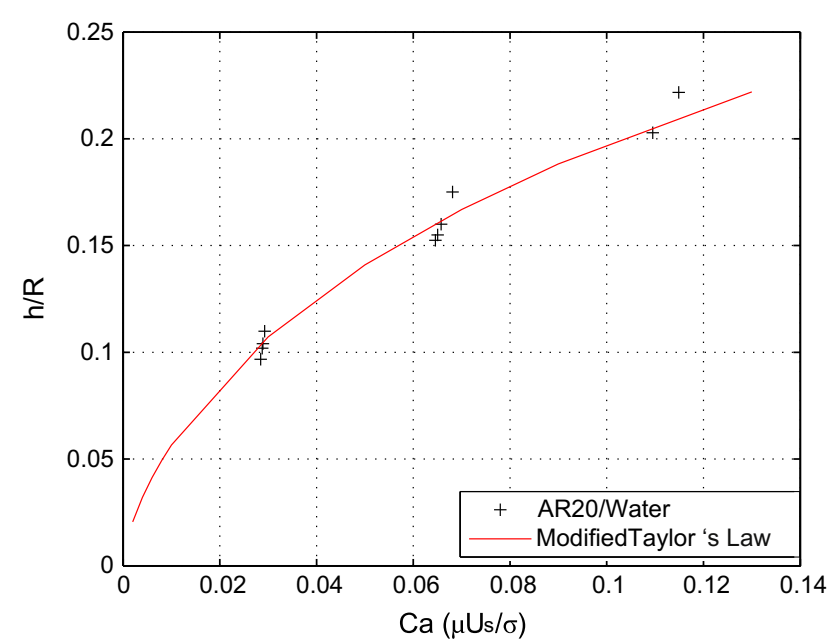

Fig. 9. Non-dimensional film thickness, $h / R$, of AR20/water flows plotted against Capillary number based on the mean slug velocity. Included in the plot is a modified Taylor's Law.

The remaining experimental data lies in the visco-inertial regime, where Ca > Ca*. Similar to Aussillous and Quere (2000), the magnitude of the film in this regime, shown in the enlarged view in Fig. 8, is under predicted by Taylor's Law. Han and Shikazono (2009) developed an expression, presented in Table 1, to account for the increased inertial effects in visco-inertial regime by incorporating the Reynolds and Weber numbers. In a study examining film thickness in liquid-gas flow regimes, Howard and Walsh (2013) found that this expression provided a reasonable approximation of the magnitude of the film. However, as can be seen in the enlarged view in Fig. 8, this expression under predicts the magnitude of the film in a liquid-liquid flow regime. While there are a number of differences between liquid-liquid flows and liquid-gas flows, the primary disparity between the flow regimes is the viscosity difference that exists between the two phases. In a liquid-gas flow regime, the viscosity of the discontinuous gaseous phase can be assumed to be negligible. Consequently, the shear forces at the interface can also be assumed negligible. However, in a liquid-liquid flow regime, the reduced viscosity difference means a significant increase in the shear forces at the interface between the phases. This results in a non-negligible flow in the liquid film surrounding the aqueous slug, hence the slug velocity and the thickness of the film that encapsulates the aqueous slug differ from those encountered in liquid-gas flows. Consequently, the poor agreement between the experimental data and the models, which were developed using liquid-gas flow regimes, is unsurprising. The Han and Shikazono (2009) model, developed specifically for this type of flow regime, under predicts the magnitude of the film, by $5.3-42.5 \%$ respectively. While the Bretherton (1960) model provides the best approximation of the experimental data in this regime, however, it also under predicts the magnitude of the film, by $4.5-41.3 \%$ respectively. The comparison between the experimental data and the correlations from the literature illustrates their inapplicability for use with liquid-liquid flows in a visco-inertial regime and highlights the need for an alternative approach to estimate the magnitude of the film.

To address this void in the literature, the following approach has been developed. The experimental data in the visco-inertial regime exhibits the following trends: $\operatorname{Re} \gg 1, \mathrm{Ca} \ll 1$ and $\mathrm{We} \ll 1$. These non-dimensional groups present the forces that endeavour to exert the greatest influence on the magnitude of the film. High Reynolds, Re $>15$, and low Capillary, Ca $<0.01$, numbers indicate that the inertial and capillary forces have a greater impact than the viscous forces on the magnitude of the film. The non-dimen- sional Weber number, which presents the relationship between the inertial and capillary forces, ranges from 0.047 to 0.697 and implies that the capillary forces play a more dominant role. A regression analysis of the experimental data, using Matlab (version 2010a), was completed to ascertain which forces or, combination of forces, has the greatest influence on the thickness of the film. Regression analysis is a statistical tool that can be used to identify the form of the relationship that exists between the dependent, film thickness, and independent variables, namely the dimensionless slug length, Capillary, Reynolds and Weber numbers. The analysis highlighted the Capillary and Weber numbers as the nondimensional groups that best scaled the experimental data. The form of this relationship, presented in Eq. (9), was determined by the scaling technique used, which, due to the low residual levels, identified it as the most pertinent means of representing the data. The form of Eq. (9) presents a slight variation on those of Bretherton (1960), Fairbrother and Stubbs (1935) and Aussillous and Quere (2000), where inertial effects on film thickness are assumed negligible, and presents a much simpler expression than that of Han and Shikazono (2009), where inertial effects are accounted for through the addition of both the Reynolds and Weber numbers.

$\frac{h}{R}=\left(\mathrm{Ca}^{0.47}\right)\left(\mathrm{We}^{0.13}\right)$

$\frac{h}{R}=\frac{\rho^{0.13} D^{0.13} \mu^{0.47} U_{s}^{0.73}}{\sigma^{0.60}}$

Eq. (10) presents a breakdown of Eq. (9) into its physical constituents. This provides an insight into which physical mechanisms have the greatest influence on the thickness of the film. Similar to the expression developed by Han and Shikazono (2009)), the mean slug velocity and capillary forces exert the greatest influence on the magnitude of the film, $U_{s}^{0.73} / \sigma^{0.6}$. Eq. (9) provided a good collapse of the experimental data and further analysis of the scaled data identified that a power law trend, of the form presented in Eq. (11), accurately represented the scaled data.

$$
\begin{aligned}
\frac{h}{R} & =0.35\left((\mathrm{Ca})^{0.47}(\mathrm{We})^{0.13}\right)^{0.75} \text { or simply } \frac{h}{R} \\
& =0.35(\mathrm{Ca})^{0.354}(\mathrm{We})^{0.097}
\end{aligned}
$$

Similar to expressions put forward by Bretherton (1960), Fairbrother and Stubbs (1935) and Aussillous and Quere (2000), Eq. (11) illustrates that the Capillary number, hence the interaction between the viscous and capillary forces, has the greatest influence on the magnitude of the film. Analysis of the $\mathrm{Ca}^{*}$ and $\mathrm{Ca}$ data, listed in Table 3, shows that the experimental data has, in some cases, just entered into the visco-inertial regime. Therefore the small weighting of the Weber number, We $\mathrm{e}^{0.097}$, compared to the Capillary number, $\mathrm{Ca}^{0.354}$, is unsurprising and highlights the dependency of film thickness on Capillary number over the $\mathrm{Ca}$, We and Re ranges of $0.002-$ $0.119,0.047-0.697$ and $14.46-100.96$ respectively.

Plotted in Fig. 10 is a comparison of the experimental data with the expression presented in Eq. (11) and the most applicable correlation for flow in a visco-inertial regime, the correlation of Han and Shikazono (2009). While the expression in Eq. (11) was developed using a small number of data points, the ranges in non-dimensional parameters reflects those encountered in most applications that employ liquid-liquid slug flow regimes and Eq. (11) provides a better approximation of the data than previous models presented in the literature, within $15 \%$.

\section{Conclusions}

The current study presented novel experimental measurements on the hydrodynamics of liquid-liquid slug flow regimes. Optical 


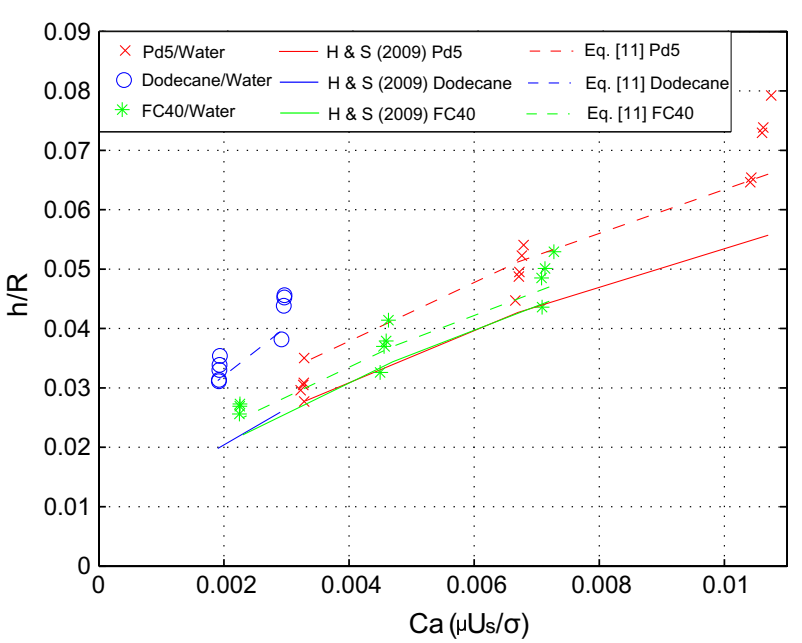

Fig. 10. Non-dimensional film thickness, $h / R$, plotted against Capillary number based on the mean slug velocity. Experimental results are compared with the correlation of Han and Shikazono (2009) and the model proposed in Eq. (11).

microscopy was used to acquire images of the film that separates the aqueous slugs from the channel wall and from these images the magnitude of the film was determined. In total, four carrier oil/water combinations were examined as part of this study; with the effects of aqueous slug length and carrier phase fluidic properties on the magnitude of the film recorded. The images revealed changes in film thickness with aqueous slug length. Below a threshold value of aqueous slug length, $L_{D}^{*} \leqslant 1.25$, the slug profile and hence film thickness varied along the entire length of the slug. As aqueous slug length increased, $L_{D}^{*} \geqslant 1.25$, distinct transition regions emerged between the front and rear slug caps. However, above an upper threshold value, $L_{D}^{*} \geqslant 1.86$, a uniform film was found to be present along the aqueous slug over a specific length.

Analysis of the experimental data illustrated the dependency of film thickness in liquid-liquid slug flows on Capillary number. This was particularly evident for data in the visco-capillary regime, where film thickness showed a sole dependency on Capillary number and a modified Taylor's Law is presented that provides excellent agreement with the experimental data. However, once the flow transitioned into the visco-inertial regime, film thickness was no longer solely dependent on Capillary number and an expression is presented that accounts for the increased inertial effects by incorporating the Weber number.

Overall, the findings reported herein provide a greater understanding of parameters that affect film thickness in liquid-liquid slug flow regimes and expressions are proposed that can be used for predictive purposes to estimate its magnitude.

\section{Acknowledgments}

The authors gratefully acknowledge the financial support of the Irish Research Council through the Embark Initiative. The involve- ment of Jeff Punch is supported by Science Foundation Ireland under Grant No. 10/CE/I1853.

\section{References}

Araujo, J.D.P., Miranda, J.M., Pinto, A.M.F.R., Campos, J.B.L.M., 2012. Wide ranging survey on the laminar flow of individual taylor bubbles rising through stagnant newtonian liquids. International Journal of Multiphase Flow 43, 131-148.

Aussillous, P., Quere, D., 2000. Quick deposition of a fluid on the wall of a tube. Physics of Fluids 12, 2367-2371.

Bretherton, F.P., 1960. The motion of long bubbles in tubes. Journal of Fluid Mechanics 10, 166-188.

Curran, K., Colin, S., Baldas, L., Davies, M., 2005. Liquid bridge instability applied to microfluidics. Microfluidics and Nanofluidics 1, 336-345.

Fairbrother, F., Stubbs, A.E., 1935. Studies in electroendosmosis: the bubble method of measurement. Journal of the Chemical Society 1, 527-529.

Ghaini, A., Kashid, M.N., Agar, D.W., 2010. Effective interfacial area for mass transfer in the liquid-liquid slug flow microreactors. Chemical Engineering and Processing 49, 358-366.

Giavedoni, M.D., Saita, F.A., 1999. The rear meniscus of a long bubble steadily displacing a newtonian liquid in a capillary tube. Physics of Fluids 11, 786.

Goldsmith, H.L., Mason, S.G., 1963. The flow of suspensions through tubes - II single large bubbles. Journal of Colloid and Interface Science 18, 237-261.

Grimes, R., King, C., Walsh, E., 2007. Film thickness for two phase flow in a microchannel. Advances and Applications in Fluid Mechanics 2, 59-70.

Han, Y., Shikazono, N., 2009. Measurement of the liquid film thickness in micro tube slug flow. International Journal of Heat and Fluid Flow 30, 842-853.

Heil, M., 2001. Finite Reynolds number effects in the Bretherton problem. Physics of Fluids 13, 2517-2521.

Howard, J.A., Walsh, P.A., 2013. Review and extensions to film thickness and bubble drift velocity prediction methods in laminar taylor or slug flows. International Journal of Multiphase Flow 55, 32-42.

Howard, J.A., Walsh, P.A., Walsh, E.J., 2011. Prandtl and capillary effects on heat transfer performance within laminar liquid-gas slug flows. International Journal of Heat and Mass Transfer 54, 4752-4761.

Irandoust, S., Andersson, B., 1989. Liquid film in Taylor flow through a capillary. Industrial and Engineering Chemical Research 28, 1684-1688.

Jovanovic, J., Zhou, W., Rebrov, E.V., Nijhuis, T.A., 2010. Liquid-liquid slug flow: hydrodynamics and pressure drop. Chemical Engineering Science 66, 42-54.

Kashid, M.N., Gerlach, I., Goetz, S., Franzke, F., Acker, J.F., Agar, D.W., Turek, S., 2005. Internal circulation within the liquid slugs of a liquid-liquid slug flow capillary microreactor. Industrial and Engineering Chemical Research 44, 5003-5010.

Kline, S.J., McClintock, F.A., 1953. Describing uncertainties in single sample experiments. Mechanical Engineering 75, 385-387.

Kreutzer, M.T., Kaptejin, F., Moulijn, J.A., Heiszwolf, J.J., 2005. Multiphase monolith reactors: chemical reaction engineering of segmented flow in microchannels. Chemical Engineering Science 60, 5895-5916.

Martinez, M.J., Undell, K.S., 1990. Axisymmetric creeping motion of drops through circular tubes. Journal of Fluid Mechanics 210, 565.

Meinhart, C.D., Weresley, S.T., Santiago, J.G., 1999. PIV measurements of a microchannel flow. Experiments in Fluids 27, 414-419.

Olbricht, W.L., Kung, D.M., 1992. The deformation and breakup of liquid drops in low Reynolds number flow through a capillary. Physics of Fluids 4, 1347-1354.

Saad, S.M.I., Policova, Z., Neumann, W., 2011. Design and accuracy of pendant drop methods for surface tension measurements. Colloids and Surfaces: Physiochemical and Engineering Aspects 384, 442-452.

Taha, T., Cui, Z.F., 2004. Hydrodynamics of slug flow inside capillaries. Chemical Engineering Science 59, 1181-1190.

Taylor, G., 1960. Deposition of a viscous fluid on the wall of a tube. Journal of Fluid Mechanics 10, 161-165.

Tice, J.D., Song, H., Lyon, A.D., Ismagilov, R.F., 2003. Formation of droplets and mixing in multiphase microfluidics at low values of the Reynolds and the capillary numbers. Langmuir 19, 9127-9133. 